Portland State University

PDXScholar

Electrical and Computer Engineering Faculty

Publications and Presentations

4-1-1997

\title{
Rate-Equation Approximations in High-Gain Lasers
}

Lee W. Casperson

Portland State University

Follow this and additional works at: https://pdxscholar.library.pdx.edu/ece_fac

Part of the Electrical and Computer Engineering Commons

Let us know how access to this document benefits you.

\section{Citation Details}

Casperson, L. W. (1997). Rate-equation approximations in high-gain lasers. 55 (4), 3073-3085.

This Article is brought to you for free and open access. It has been accepted for inclusion in Electrical and Computer Engineering Faculty Publications and Presentations by an authorized administrator of PDXScholar. Please contact us if we can make this document more accessible: pdxscholar@pdx.edu. 


\title{
Rate-equation approximations in high-gain lasers
}

\author{
Lee W. Casperson* \\ The Rochester Theory Center for Optical Science and Engineering and The Institute of Optics, University of Rochester, \\ Rochester, New York 14627-0186
}

(Received 17 October 1996)

\begin{abstract}
The rate-equation approximation is one of the most fundamental and universally employed simplifications in laser analyses. The accuracy and regions of applicability of this approximation are explored in comparisons with more rigorous semiclassical models. Higher-order rate-equation approximations are also developed, and these improved models can yield much better accuracy than conventional rate equations with little added complexity. The modified adiabatic elimination methods reported here would also be useful in reducing the mathematical models governing other physical systems. [S1050-2947(97)08303-0]

PACS number(s): 42.60.Lh, 42.55.Ah, 42.50.Gy
\end{abstract}

\section{INTRODUCTION}

Every analysis of laser behavior involves approximations. One approximation that is included implicitly or explicitly in most studies of laser amplification and oscillation has come to be known as the rate-equation approximation. In this simplification the atoms or molecules are described entirely in terms of the populations of their various energy levels, and the electromagnetic fields are described entirely in terms of photon densities or intensities. In interactions between light and atoms the level populations increase or decrease while the photon numbers also change in such a way that energy is conserved. The rate of change of these populations and densities is governed by a set of first-order differential equations that are referred to as rate equations.

In the rate equations, the physical processes that couple the atomic populations to the intensities usually have very simple forms and are identified as spontaneous emission, stimulated emission, and absorption. In their most basic form, these equations were established by Einstein in 1916 and were employed by him in deriving a fundamental relationship between the spontaneous- and stimulated-emission rate coefficients [1]. With the invention of lasers, many other uses for the rate equations have been developed. Among the earliest rate-equation studies of laser oscillators were the analyses by Statz and DeMars [2] and Dunsmuir [3]. These authors were interested in the pulsations and relaxation oscillations that occur when there is a sudden change in any of the parameters governing otherwise steady-state laser oscillations. Their studies and many that followed focused on systems in which the populations and intensities may vary in time but not in space. Among the earliest rate-equation studies of laser amplifiers were the treatments by Wright and Schulz-DuBois [4] and by Rigrod [5]. Their studies emphasized systems in which the populations and intensities may vary in space but not in time. In addition, there have also been numerous rate-equation studies of laser systems in which both space and time effects are included. Such models are essential for the treatment of pulse evolution in saturating

\footnotetext{
*Permanent address: Department of Electrical Engineering, Portland State University, P.O. Box 751, Portland, OR 97207-0751.
}

laser systems, and early treatments were those of Bellman, Birnbaum, and Wagner [6], Frantz and Nodvik [7], and Schulz-DuBois [8]. Later studies of these topics have included more complicated energy-level structures and geometries, but most are still basically rate-equation formulations.

Laser systems can also be analyzed using semiclassical models. In these models the atomic behavior is based more directly on Schrödinger's equation (or Dirac's equation), while the electromagnetic fields are tied more closely to the Maxwell-Heaviside equations. Early semiclassical models for the dynamics of homogeneously broadened maser oscillators were reported by Gurtovnik [9] and others, and a detailed semiclassical analysis of homogeneously broadened lasers was given by Tang [10]. The first comprehensive semiclassical development for inhomogeneously broadened lasers was by Lamb [11]. In principle, such semiclassical models are able to represent atomic and electromagnetic wave functions having arbitrary polarizations and arbitrarily fast variations in time and space. In practice, even semiclassical treatments typically incorporate approximations which may significantly restrict their range of applicability. These models do, however, retain information about the phases of the atomic wave functions and the electromagnetic fields, and it is exactly this phase information that is eliminated by the rate-equation approximation. Within the rate-equation approximation, the only dynamical variables are generally the real amplitudes of the wave functions (corresponding to probability densities) and the fields (corresponding to photon densities or intensities).

The usual reason for employing the rate-equation approximation when more rigorous models are available is to achieve greater analytical simplicity. The rate-equation models have fewer equations and simpler variables, and solution methods are always more straightforward. Explicit analytical solutions can often be obtained, and in any case numerical solutions are much less complicated. Thus, it is a matter of considerable practical importance to know the conditions under which the rate-equation models may be sufficiently accurate for a particular application. A major purpose of this study is to examine the limits of the rate-equation approximation, emphasizing its first and most basic application to transients in laser oscillators. For this application one finds that the rate-equation approximations are most likely to fail 
in lasers employing very high-gain-amplifying media; and semiclassical spontaneous pulsations, for example, have only been observed in high-gain lasers.

It is shown here that there is actually a hierarchy of rateequation approximations, and many simplifying advantages of the rate-equation approach can be retained while increasing substantially the accuracy of the overall results. Thus, in the higher-order rate-equation approximations, the total number of equations is still reduced from the semiclassical models while the variables are the same simple scalar quantities used in the more standard rate equations. The only penalty of the new higher-order results appears in the form of extra terms on the right-hand side of the first-order differential equations. For some regions of operation, these new rate equations are found to have significant advantages. The modified adiabatic elimination methods employed here would also be useful in reducing the mathematical models governing other physical systems.

A general semiclassical model is reviewed in Sec. II for the dynamics of a laser having arbitrary levels of homogeneous and inhomogeneous line broadening. Use of this starting point provides a common basis for this analysis and previous investigations of laser instabilities [12], a frequency approximation [13], time- [14], and space- [15] derivative approximations, and the rotating-wave approximation [16]. The reduction of the semiclassical laser equations to simpler rate-equation sets is discussed in Sec. III, and a detailed numerical comparison of the various approximations is included in Sec. IV. For small perturbations away from the steady state, linearized solutions are useful; an investigation of the linearized versions of the models is included in Sec. V.

\section{GENERAL MODEL}

The starting point for this study is the same basic onedimensional semiclassical model that has been employed in Refs. [12-16] and elsewhere. The density-matrix equations in this model take the form

$$
\begin{aligned}
\left(\frac{\partial}{\partial t}+v \frac{\partial}{\partial z}\right) \rho_{a b}\left(v, \omega_{\alpha}, z, t\right)= & -\left(i \omega_{\alpha}+\gamma\right) \rho_{a b}\left(v, \omega_{\alpha}, z, t\right) \\
& -\frac{i \mu}{\hbar} E(z, t)\left[\rho_{a a}\left(v, \omega_{\alpha}, z, t\right)\right. \\
& \left.-\rho_{b b}\left(v, \omega_{\alpha}, z, t\right)\right]
\end{aligned}
$$

$$
\begin{aligned}
\left(\frac{\partial}{\partial t}+\right. & \left.v \frac{\partial}{\partial z}\right) \rho_{a a}\left(v, \omega_{\alpha}, z, t\right) \\
= & \lambda_{a}\left(v, \omega_{\alpha}, z, t\right)-\gamma_{a} \rho_{a a}\left(v, \omega_{\alpha}, z, t\right) \\
& +\left[\frac{i \mu}{\hbar} E(z, t) \rho_{b a}\left(v, \omega_{\alpha}, z, t\right)+\text { c.c. }\right],
\end{aligned}
$$

$$
\begin{aligned}
&\left(\frac{\partial}{\partial t}+v\right.\left.\frac{\partial}{\partial z}\right) \rho_{b b}\left(v, \omega_{\alpha}, z, t\right) \\
&= \lambda_{b}\left(v, \omega_{\alpha}, z, t\right)-\gamma_{b} \rho_{b b}\left(v, \omega_{\alpha}, z, t\right) \\
&+\gamma_{a b} \rho_{a a}\left(v, \omega_{\alpha}, z, t\right) \\
&-\left[\frac{i \mu}{\hbar} E(z, t) \rho_{b a}\left(v, \omega_{\alpha}, z, t\right)+\text { c.c. }\right], \\
& \rho_{b a}\left(v, \omega_{\alpha}, z, t\right)=\rho_{a b}^{*}\left(v, \omega_{\alpha}, z, t\right),
\end{aligned}
$$

where the subscripts $a$ and $b$ denote the upper and lower laser levels, respectively, $\gamma_{a}$ and $\gamma_{b}$ are the total decay rates of these levels, $\gamma_{a b}$ is the rate of direct decays from level $a$ to level $b, \gamma$ is the decay rate of the off-diagonal elements, $\lambda_{a}$ and $\lambda_{b}$ are the pumping rates, $\mu$ is the dipole moment of the laser transition, and the notation c.c. means the complex conjugate of the preceding terms. The laser medium is assumed to have both Doppler and non-Doppler broadening mechanisms, with $v$ being the $z$ component of the velocity and $\omega_{\alpha}$ the center frequency of the laser transition for members of an atomic or molecular class $\alpha$. The decay process represented by $\gamma_{a b}$ is often missing from theoretical studies, but it can have an important effect on the population of the lower level of the laser transition.

To the density-matrix equations for the atomic or molecular populations and polarizations must be added an equation for the electric field. The wave equation for the electric field of a linearly polarized wave in a laser medium can be written

$$
\frac{\partial^{2} E(z, t)}{\partial z^{2}}-\mu_{1} \sigma \frac{\partial E(z, t)}{\partial t}-\mu_{1} \varepsilon_{1} \frac{\partial^{2} E(z, t)}{\partial t^{2}}=\mu_{1} \frac{\partial^{2} P(z, t)}{\partial t^{2}} .
$$

The permeability $\mu_{1}$ and permittivity $\varepsilon_{1}$ should be understood to include all of the magnetic and dielectric properties of the laser medium except for the polarization $P(z, t)$, which is due to the lasing atoms or molecules. The polarization driving this equation can be related back to the offdiagonal density matrix elements by

$$
P(z, t)=\int_{0}^{\infty} \int_{-\infty}^{\infty} \mu \rho_{a b}\left(v, \omega_{\alpha}, z, t\right) d v d \omega_{\alpha}+\text { c.c. }
$$

Equations (1)-(6) are a complete set from which the time and space dependences of the electric field and of the atomic or molecular parameters can be determined, subject to the boundary conditions at the resonator mirrors.

The simplest dynamical solutions for the model described by Eqs. (1)-(6) are those that apply to uniformly pumped one-directional ring lasers, in which both the electric field and the polarization are traveling waves. If the gain and loss in a ring laser can be considered to be uniformly distributed over a completely filled cavity, then the rapid time and space variations in the model can be factored out by means of the substitutions

$$
E(z, t)=(1 / 2) E^{\prime}(t) \exp (i k z-i \omega t)+\text { c.c. },
$$




$$
\rho_{a b}\left(v, \omega_{\alpha}, z, t\right)=P^{\prime}\left(v, \omega_{\alpha}, t\right) \exp (i k z-i \omega t) / 2 \mu .
$$

With the usual rotating-wave approximation, these substitutions lead to the new spatially independent set

$$
\begin{aligned}
\frac{\partial P^{\prime}\left(v, \omega_{\alpha}, t\right)}{\partial t}= & i\left(\omega-\omega_{\alpha}-k v\right) P^{\prime}\left(v, \omega_{\alpha}, t\right)-\gamma P^{\prime}\left(v, \omega_{\alpha}, t\right) \\
& -\frac{i \mu^{2}}{\hbar} E^{\prime}(t)\left[\rho_{a a}\left(v, \omega_{\alpha}, t\right)-\rho_{b b}\left(v, \omega_{\alpha}, t\right)\right],
\end{aligned}
$$

$$
\begin{aligned}
& \frac{\partial \rho_{a a}\left(v, \omega_{\alpha}, t\right)}{\partial t}=\lambda_{a}\left(v, \omega_{\alpha}, t\right)-\gamma_{a} \rho_{a a}\left(v, \omega_{\alpha}, t\right) \\
& +\frac{i}{4 \hbar}\left[E^{\prime}(t) P^{\prime *}\left(v, \omega_{\alpha}, t\right)\right. \\
& \left.-E^{\prime *}(t) P^{\prime}\left(v, \omega_{\alpha}, t\right)\right], \\
& \frac{\partial \rho_{b b}\left(v, \omega_{\alpha}, t\right)}{\partial t}=\lambda_{b}\left(v, \omega_{\alpha}, t\right)-\gamma_{b} \rho_{b b}\left(v, \omega_{\alpha}, t\right) \\
& +\gamma_{a b} \rho_{a a}\left(v, \omega_{\alpha}, t\right) \\
& -\frac{i}{4 \hbar}\left[E^{\prime}(t) P^{\prime *}\left(v, \omega_{\alpha}, t\right)\right. \\
& \left.-E^{\prime *}(t) P^{\prime}\left(v, \omega_{\alpha}, t\right)\right], \\
& \frac{d^{2} E^{\prime}(t)}{d t^{2}}+\left(\frac{\sigma}{\varepsilon_{1}}-2 i \omega\right) \frac{d E^{\prime}(t)}{d t}-\left(\omega^{2}-\Omega^{2}+i \frac{\sigma}{\varepsilon_{1}} \omega\right) E^{\prime}(t) \\
& =-\frac{1}{\varepsilon_{1}}\left(\frac{\partial^{2}}{\partial t^{2}} \int_{0}^{\infty} \int_{-\infty}^{\infty} P^{\prime}\left(v, \omega_{\alpha}, t\right) d v d \omega_{\alpha}\right. \\
& -2 i \omega \frac{\partial}{\partial t} \int_{0}^{\infty} \int_{-\infty}^{\infty} P^{\prime}\left(v, \omega_{\alpha}, t\right) d v d \omega_{\alpha} \\
& \left.-\omega^{2} \int_{0}^{\infty} \int_{-\infty}^{\infty} P^{\prime}\left(v, \omega_{\alpha}, t\right) d v d \omega_{\alpha}\right)
\end{aligned}
$$

where the new frequency $\Omega=k\left(\mu_{1} \varepsilon_{1}\right)^{-1 / 2}$ has been introduced, and the conductivity $\sigma$ is understood to represent all cavity losses. The limitations of the rotating-wave approximation have been explored in Ref. [16].

It is now usual to drop some of the higher derivative terms in the field equation by arguing that the field and polarization envelopes vary negligibly in an optical cycle and that the losses per optical cycle are also negligible. The terms that remain are

$$
\begin{aligned}
\frac{d E^{\prime}(t)}{d t}= & -\gamma_{c} E^{\prime}(t)+i \frac{\left(\omega^{2}-\Omega^{2}\right)}{2 \omega} E^{\prime}(t) \\
& +i \frac{\omega}{2 \varepsilon_{1}} \int_{0}^{\infty} \int_{-\infty}^{\infty} P^{\prime}\left(v, \omega_{\alpha}, t\right) d v d \omega_{\alpha}
\end{aligned}
$$

where the losses are represented by the field decay rate $\gamma_{c}=\sigma / 2 \varepsilon_{1}$. Within this approximation the frequency $\Omega$ can now be recognized as the nondispersed cavity resonance fre- quency, i.e., the lasing frequency that would result if the dispersion or real part of the polarization $P^{\prime}\left(v, \omega_{\alpha}, t\right)$ were equal to zero. The consequences and limitations of this derivative approximation have been considered in Ref. [14].

Further frequency approximations are also commonly introduced. If the lasing frequency $\omega$ is close to the nondispersed frequency $\Omega$, then the term $\left(\omega^{2}-\Omega^{2}\right) / 2 \omega$ can be approximated by $\omega-\Omega$; if the lasing frequency is also close to a characteristic frequency of the laser transition $\omega_{0}$, then the $\omega$ multiplying the polarization integrals may be replaced by $\omega_{0}$. With these approximations, Eq. (10) reduces to

$$
\begin{aligned}
\frac{d E^{\prime}(t)}{d t}= & -\gamma_{c} E^{\prime}(t)+i(\omega-\Omega) E^{\prime}(t) \\
& +i \frac{\omega_{0}}{2 \varepsilon_{1}} \int_{0}^{\infty} \int_{-\infty}^{\infty} P^{\prime}\left(v, \omega_{\alpha}, t\right) d v d \omega_{\alpha}
\end{aligned}
$$

This result appears in one form or another in most semiclassical laser models. The limitations on the validity of the frequency approximations for steady-state laser behavior have been considered in Ref. [13].

The polarization amplitude $P^{\prime}\left(v, \omega_{\alpha}, t\right)$ and the field amplitude $E^{\prime}(t)$ appearing in Eqs. (9)-(11) and (14) are complex quantities. It is helpful to separate them into their real and imaginary parts in the forms $E^{\prime}(t)=E_{r}(t)+i E_{i}(t)$ and $P^{\prime}\left(v, \omega_{\alpha}, t\right)=P_{r}\left(v, \omega_{\alpha}, t\right)+i P_{i}\left(v, \omega_{\alpha}, t\right)$. With these substitutions the model becomes

$$
\begin{aligned}
\frac{\partial P_{r}\left(v, \omega_{\alpha}, t\right)}{\partial t}= & -\left(\omega-\omega_{\alpha}-k v\right) P_{i}\left(v, \omega_{\alpha}, t\right)-\gamma P_{r}\left(v, \omega_{\alpha}, t\right) \\
& +\frac{\mu^{2}}{\hbar} E_{i}(t) D\left(v, \omega_{\alpha}, t\right)
\end{aligned}
$$

$$
\begin{aligned}
\frac{\partial P_{i}\left(v, \omega_{\alpha}, t\right)}{\partial t}= & \left(\omega-\omega_{\alpha}-k v\right) P_{r}\left(v, \omega_{\alpha}, t\right)-\gamma P_{i}\left(v, \omega_{\alpha}, t\right) \\
& -\frac{\mu^{2}}{\hbar} E_{r}(t) D\left(v, \omega_{\alpha}, t\right)
\end{aligned}
$$

$$
\begin{aligned}
\frac{\partial D\left(v, \omega_{\alpha}, t\right)}{\partial t}= & \lambda_{a}\left(v, \omega_{\alpha}, t\right)-\lambda_{b}\left(v, \omega_{\alpha}, t\right) \\
& -\frac{\gamma_{a}+\gamma_{a b}+\gamma_{b}}{2} D\left(v, \omega_{\alpha}, t\right) \\
& -\frac{\gamma_{a}+\gamma_{a b}-\gamma_{b}}{2} M\left(v, \omega_{\alpha}, t\right) \\
& +\frac{1}{\hbar}\left[E_{r}(t) P_{i}\left(v, \omega_{\alpha}, t\right)-E_{i}(t) P_{r}\left(v, \omega_{\alpha}, t\right)\right],
\end{aligned}
$$




$$
\begin{aligned}
\frac{\partial M\left(v, \omega_{\alpha}, t\right)}{\partial t}= & \lambda_{a}\left(v, \omega_{\alpha}, t\right)+\lambda_{b}\left(v, \omega_{\alpha}, t\right) \\
& -\frac{\gamma_{a}-\gamma_{a b}-\gamma_{b}}{2} D\left(v, \omega_{\alpha}, t\right) \\
& -\frac{\gamma_{a}-\gamma_{a b}+\gamma_{b}}{2} M\left(v, \omega_{\alpha}, t\right),
\end{aligned}
$$

$$
\begin{aligned}
\frac{d E_{r}(t)}{d t}= & -\gamma_{c} E_{r}(t)-(\omega-\Omega) E_{i}(t) \\
& -\frac{\omega_{0}}{2 \varepsilon_{1}} \int_{0}^{\infty} \int_{-\infty}^{\infty} P_{i}\left(v, \omega_{\alpha}, t\right) d v d \omega_{\alpha},
\end{aligned}
$$$$
\frac{d E_{i}(t)}{d t}=-\gamma_{c} E_{i}(t)+(\omega-\Omega) E_{r}(t)
$$$$
+\frac{\omega_{0}}{2 \varepsilon_{1}} \int_{0}^{\infty} \int_{-\infty}^{\infty} P_{r}\left(v, \omega_{\alpha}, t\right) d v d \omega_{\alpha},
$$

where we have also introduced the population difference $D\left(v, \omega_{\alpha}, t\right)=\rho_{a a}\left(v, \omega_{\alpha}, t\right)-\rho_{b b}\left(v, \omega_{\alpha}, t\right)$ and sum $M\left(v, \omega_{\alpha}, t\right)=\rho_{a a}\left(v, \omega_{\alpha}, t\right)+\rho_{b b}\left(v, \omega_{\alpha}, t\right)$. Equations (15)(20) summarize an established model for the dynamics of laser oscillators, and they are applicable to a wide range of transient phenomena such as instabilities, the giant pulsations arising from gain or loss switching, and damped relaxation oscillations as a laser returns to $\mathrm{cw}$ operation. While they appear as six equations, there are of course many times more equations, depending on the way in which the inhomogeneous broadening must be represented by multiple values of the population and polarization variables. These equations will serve as our starting point for discussions of the rateequation approximation.

The basic idea behind the usual or zeroth-order rateequation approximation is that in many laser systems neither the electric field nor the atomic populations vary significantly within the coherence time of the atomic wave functions. In this case, the polarization amplitudes $P_{r}\left(v, \omega_{\alpha}, t\right)$ and $P_{i}\left(v, \omega_{\alpha}, t\right)$, which decay according to the coherence decay rate, will always be instantaneous functions of the fields and populations; and the derivatives in the polarization equations [Eqs. (15) and (16)] can be set equal to zero. This assignment eliminates two differential equations from the mathematical model, and in some cases this substantial simplification even makes possible analytical solutions. On the other hand, it is sometimes the case that the electric field and populations do change significantly during the coherence time, and the rate-equation approximation would then seem to be inapplicable. We will find in the following investigation that it is sometimes helpful to employ a new higherorder rate-equation approximation for those circumstances where the coherence decay rate is large but not infinite. In this case, elimination of the polarization differential equation is still possible, but as noted above additional terms may be necessary in the remaining equations. This approach will be outlined here for the general model that has been developed above, and it will be explored in detail in the following sections for the simpler special case of a laser having only homogeneous line broadening.

Equations (15) and (16) can be interpreted as ordinary linear first-order differential equations with nonconstant coefficients. Thus, their formal solutions can be written as [17]

$$
\begin{aligned}
P_{r}\left(v, \omega_{\alpha}, t\right)= & \int_{-\infty}^{t} \exp \left[\gamma\left(t^{\prime}-t\right)\right] \\
& \times\left[-\left(\omega-\omega_{\alpha}-k v\right) P_{i}\left(v, \omega_{\alpha}, t^{\prime}\right)\right. \\
& \left.+\frac{\mu^{2}}{\hbar} E_{i}\left(t^{\prime}\right) D\left(v, \omega_{\alpha}, t^{\prime}\right)\right] d t^{\prime}, \\
P_{i}\left(v, \omega_{\alpha}, t\right)= & \int_{-\infty}^{t} \exp \left[\gamma\left(t^{\prime}-t\right)\right] \\
& \times\left[\left(\omega-\omega_{\alpha}-k v\right) P_{r}\left(v, \omega_{\alpha}, t^{\prime}\right)\right. \\
& \left.-\frac{\mu^{2}}{\hbar} E_{r}\left(t^{\prime}\right) D\left(v, \omega_{\alpha}, t^{\prime}\right)\right] d t^{\prime},
\end{aligned}
$$

where it is assumed that the polarization components vanish at the initial time $t=-\infty$. These are, of course, not complete solutions and have only served to transform the differential equations into integral equations. For large values of the coherence decay rate $\gamma$, however, the exponential functions inside of the integrands act like Dirac $\delta$ functions. Thus, the values of the other functions within the integrand are only important for times within about $\gamma^{-1}$ of the time $t$. If those functions vary negligibly within that time, they may be removed from the integral after evaluation at the time $t$ of the $\delta$-function maximum. The results of this simplification are

$$
\begin{aligned}
P_{r}\left(v, \omega_{\alpha}, t\right)= & -\left(\omega-\omega_{\alpha}-k v\right) P_{i}\left(v, \omega_{\alpha}, t\right)+\frac{\mu^{2}}{\hbar} \\
& \left.\times E_{i}(t) D\left(v, \omega_{\alpha}, t\right)\right] \int_{-\infty}^{t} \exp \left[\gamma\left(t^{\prime}-t\right)\right] d t^{\prime} \\
= & \frac{1}{\gamma}\left[-\left(\omega-\omega_{\alpha}-k v\right) P_{i}\left(v, \omega_{\alpha}, t\right)\right. \\
& \left.+\frac{\mu^{2}}{\hbar} E_{i}(t) D\left(v, \omega_{\alpha}, t\right)\right], \\
P_{i}\left(v, \omega_{\alpha}, t\right)= & \left(\omega-\omega_{\alpha}-k v\right) P_{r}\left(v, \omega_{\alpha}, t\right)-\frac{\mu^{2}}{\hbar} \\
& \left.\left.\times E_{r}(t) D\left(v, \omega_{\alpha}, t\right)\right]\right]_{-\infty}^{t} \exp \left[\gamma\left(t^{\prime}-t\right)\right] d t^{\prime} \\
= & \frac{1}{\gamma}\left[\left(\omega-\omega_{\alpha}-k v\right) P_{r}\left(v, \omega_{\alpha}, t\right)\right. \\
& \left.-\frac{\mu^{2}}{\hbar} E_{r}(t) D\left(v, \omega_{\alpha}, t\right)\right] .
\end{aligned}
$$


Equations (23) and (24) represent the usual rate-equation approximation and, as suggested above, the same results would be obtained by simply dropping the derivatives in Eqs. (15) and (16). The reasons for the more formal approach used here is that it will lead naturally to more accurate approximations.

The process just described may be viewed as the elimination of a differential equation from a physical model on the basis that its associated relaxation rate is fast compared to other time dependences in the model. This sort of reduction is sometimes referred to as adiabatic elimination, and it has been discussed in many places. In the laser context it has been examined in detail, for example, in Ref. [18]. In the following discussion we consider a possible improvement of the adiabatic elimination method that might be considered an adaptation of Laplace's method for approximating integrals [19].

Before proceeding with our discussion of improved rateequation models, it may be appropriate to emphasize the mathematical simplifications that result from the lowestorder rate-equation approximation that has just been made. In particular, Eqs. (23) and (24) can now be solved explicitly for the real and imaginary components of the polarization, and the results are

$$
\begin{aligned}
P_{r}\left(v, \omega_{\alpha}, t\right)= & \frac{\mu^{2} D\left(v, \omega_{\alpha}, t\right)}{\gamma \hbar} \\
& \times \frac{\left[\left(\omega-\omega_{\alpha}-k v\right) / \gamma\right] E_{r}(t)+E_{i}(t)}{1+\left[\left(\omega-\omega_{\alpha}-k v\right) / \gamma\right]^{2}}, \\
P_{i}\left(v, \omega_{\alpha}, t\right)= & \frac{\mu^{2} D\left(v, \omega_{\alpha}, t\right)}{\gamma \hbar} \\
& \times \frac{\left[\left(\omega-\omega_{\alpha}-k v\right) / \gamma\right] E_{i}(t)-E_{r}(t)}{1+\left[\left(\omega-\omega_{\alpha}-k v\right) / \gamma\right]^{2}} .
\end{aligned}
$$

If Eqs. (25) and (26) are substituted into Eqs. (17)-(20), one finds that the governing model for the laser dynamics consists of four equations rather than the previous six:

$$
\begin{aligned}
\frac{\partial D\left(v, \omega_{\alpha}, t\right)}{\partial t}= & \lambda_{a}\left(v, \omega_{\alpha}, t\right)-\lambda_{b}\left(v, \omega_{\alpha}, t\right) \\
& -\frac{\gamma_{a}+\gamma_{a b}+\gamma_{b}}{2} D\left(v, \omega_{\alpha}, t\right) \\
& -\frac{\gamma_{a}+\gamma_{a b}-\gamma_{b}}{2} M\left(v, \omega_{\alpha}, t\right) \\
& -\frac{\mu^{2} D\left(v, \omega_{\alpha}, t\right)}{\gamma \hbar^{2}} \frac{E_{r}^{2}(t)+E_{i}^{2}(t)}{1+\left[\left(\omega-\omega_{\alpha}-k v\right) / \gamma\right]^{2}},
\end{aligned}
$$

$$
\begin{gathered}
\frac{\partial M\left(v, \omega_{\alpha}, t\right)}{\partial t}=\lambda_{a}\left(v, \omega_{\alpha}, t\right)+\lambda_{b}\left(v, \omega_{\alpha}, t\right) \\
-\frac{\gamma_{a}-\gamma_{a b}-\gamma_{b}}{2} D\left(v, \omega_{\alpha}, t\right) \\
-\frac{\gamma_{a}-\gamma_{a b}+\gamma_{b}}{2} M\left(v, \omega_{\alpha}, t\right), \\
\begin{aligned}
\frac{d E_{r}(t)}{d t}=- & \gamma_{c} E_{r}(t)-(\omega-\Omega) E_{i}(t) \\
& -\frac{\omega_{0} \mu^{2}}{2 \varepsilon_{1} \gamma \hbar} \frac{\left[\left(\omega-\omega_{\alpha}-k v\right) / \gamma\right] E_{i}(t)-E_{r}(t)}{1+\left[\left(\omega-\omega_{\alpha}-k v\right) / \gamma\right]^{2}} \\
& \times \int_{0}^{\infty} \int_{-\infty}^{\infty} D\left(v, \omega_{\alpha}, t\right) d v d \omega_{\alpha}, \\
\frac{d E_{i}(t)}{d t}= & -\gamma_{c} E_{i}(t)+(\omega-\Omega) E_{r}(t) \\
& +\frac{\omega_{0} \mu^{2}}{2 \varepsilon_{1} \gamma \hbar} \frac{\left[\left(\omega-\omega_{\alpha}-k v\right) / \gamma\right] E_{r}(t)+E_{i}(t)}{1+\left[\left(\omega-\omega_{\alpha}-k v\right) / \gamma\right]^{2}} \\
& \times \int_{0}^{\infty} \int_{-\infty}^{\infty} D\left(v, \omega_{\alpha}, t\right) d v d \omega_{\alpha} .
\end{aligned}
\end{gathered}
$$

It may be noted that with the elimination of the polarizations from Eqs. (27)-(28), all information about the phase of the atomic wave functions has been lost. If Eq. (29) is multiplied by $2 E_{r}(t)$ and Eq. (30) is multiplied by $2 E_{i}(t)$ and the results are added, one obtains the result

$$
\begin{aligned}
\frac{d\left[E_{r}^{2}(t)+E_{i}^{2}(t)\right]}{d t}= & -2 \gamma_{c}\left[E_{r}^{2}(t)+E_{i}^{2}(t)\right] \\
& +\frac{\omega_{0} \mu^{2}}{\varepsilon_{1} \gamma \hbar} \frac{E_{r}^{2}(t)+E_{i}^{2}(t)}{1+\left[\left(\omega-\omega_{\alpha}-k v\right) / \gamma\right]^{2}} \\
& \times \int_{0}^{\infty} \int_{-\infty}^{\infty} D\left(v, \omega_{\alpha}, t\right) d v d \omega_{\alpha} .
\end{aligned}
$$

From Eqs. (27), (28), and (30) it is clear that the phase information concerning the fields has no effect on the populations or the intensity. In short, the rate-equation approximation reduced the original six equation set to only three equations. It is such simplifications that are the motivation for employing this approximation.

The key step in obtaining the rate-equation approximation was being able to remove the slowly varying laser parameters from the integrals in Eqs. (21) and (22). If these parameters vary significantly within a time of $\gamma^{-1}$, the rateequation approximation cannot be justified. On the other hand, this method of developing the approximation also leads to the possibility of obtaining better approximations that might be valid for moderate parameter variations within the time $\gamma^{-1}$. In particular, it is very useful to expand the parameters in the integrands of Eqs. (21) and (22) in a Taylor series about the time $t$. This procedure introduces derivatives of the parameters within the integrals, but at least for the 
next order of accuracy these derivatives are known already from the other equations in the model. With the introduction of these equations, the integrals can again be performed analytically; and the results are a new set of rate equations with much better accuracy than the usual zeroth-order set. To minimize the mathematical clutter, this procedure is illustrated in detail in the next section for the simplest special case of a homogeneously broadened laser. The generalization to other laser models of the present section or to other physical models found elsewhere will be seen to be straightforward.

\section{HOMOGENEOUS BROADENING}

For a more detailed discussion of the rate-equation approximation, it is helpful to start from a simplified version of the laser model developed in the preceding section. To be specific, we will move to the simplest possible semiclassical laser model. First, we restrict our attention to homogeneously broadened media. In this case the intrinsic center frequencies of the atoms are all identical and their velocities are zero. Thus, we replace the variable $\omega_{\alpha}$ with the constant $\omega_{0}$, and we replace $v$ with zero. Then, with a straightforward change of variables, Eqs. (15)-(20) reduce to

$$
\begin{aligned}
& \frac{d P_{r}(t)}{d t}=-\left(\omega-\omega_{0}\right) P_{i}(t)-\gamma P_{r}(t)+\frac{\mu^{2}}{\hbar} E_{i}(t) D(t), \\
& \frac{d P_{i}(t)}{d t}=\left(\omega-\omega_{0}\right) P_{r}(t)-\gamma P_{i}(t)-\frac{\mu^{2}}{\hbar} E_{r}(t) D(t), \\
& \frac{d D(t)}{d t}=\lambda_{a}(t)-\lambda_{b}(t)-\frac{\gamma_{a}+\gamma_{a b}+\gamma_{b}}{2} D(t) \\
& -\frac{\gamma_{a}+\gamma_{a b}-\gamma_{b}}{2} M(t)+\frac{1}{\hbar}\left[E_{r}(t) P_{i}(t)\right. \\
& \left.-E_{i}(t) P_{r}(t)\right], \\
& \frac{d M(t)}{d t}=\lambda_{a}(t)+\lambda_{b}(t)-\frac{\gamma_{a}-\gamma_{a b}-\gamma_{b}}{2} D(t) \\
& -\frac{\gamma_{a}-\gamma_{a b}+\gamma_{b}}{2} M(t), \\
& \frac{d E_{r}(t)}{d t}=-\gamma_{c} E_{r}(t)-(\omega-\Omega) E_{i}(t)-\frac{\omega_{0}}{2 \varepsilon_{1}} P_{i}(t), \\
& \frac{d E_{i}(t)}{d t}=-\gamma_{c} E_{i}(t)+(\omega-\Omega) E_{r}(t)+\frac{\omega_{0}}{2 \varepsilon_{1}} P_{r}(t) .
\end{aligned}
$$

The previous laser model appeared to contain six coupled differential equations, but with inhomogeneous broadening the actual number of equations was much larger. Now, in the homogeneous limit, Eqs. (32)-(37) really are just six equations.

It is also helpful to adopt a simpler energy-level scheme. For example, if $\gamma_{b}$ is set equal to $\gamma_{a}+\gamma_{a b}$, then Eq. (34) becomes independent of the parameter $M(t)$. If $\gamma_{a b}$ is small compared to $\gamma_{a}$, this condition has the interpretation that the net decay rates of the two levels are almost equal. In this case, Eq. (35) for $M(t)$ can be ignored and, with suitable redefinitions of the pumping and decay rates, Eq. (34) can be written

$$
\frac{d D(t)}{d t}=\lambda_{d}(t)-\gamma_{d} D(t)+\frac{1}{\hbar}\left[E_{r}(t) P_{i}(t)-E_{i}(t) P_{r}(t)\right] .
$$

This same form can also be obtained by assuming that the lower state remains empty. In either case the model now consists of five coupled differential equations.

Finally, we will also assume that the laser frequency and cavity resonance are at the center frequency of the atomic transition $\left(\omega=\omega_{0}=\Omega\right)$. With this restriction it is possible to set $P_{r}(t)$ and $E_{i}(t)$ equal to zero, and our general model reduces to the familiar set [10]

$$
\begin{gathered}
\frac{d P_{i}(t)}{d t}=-\gamma P_{i}(t)-\frac{\mu^{2}}{\hbar} E_{r}(t) D(t), \\
\frac{d D(t)}{d t}=\lambda_{d}(t)-\gamma_{d} D(t)+\frac{1}{\hbar} E_{r}(t) P_{i}(t), \\
\frac{d E_{r}(t)}{d t}=-\gamma_{c} E_{r}(t)-\frac{\omega_{0}}{2 \varepsilon_{1}} P_{i}(t) .
\end{gathered}
$$

For notational simplicity we use normalized forms of the dependent variables and Eqs. (39)-(41) become [20]

$$
\begin{gathered}
\frac{d P(t)}{d t}=-\gamma[P(t)+A(t) D(t)], \\
\frac{d D(t)}{d t}=-\gamma_{d}[D(t)-r(t)-A(t) P(t)], \\
\frac{d A(t)}{d t}=-\gamma_{c}[A(t)+P(t)],
\end{gathered}
$$

where $A(t)$ represents the amplitude of the electric field. The symbol $r$ represents the pump rate as a threshold parameter, and steady-state lasing is not possible if $r$ is not a constant greater than unity.

As in the preceding section, we see that Eq. (42) may be regarded as a linear first-order differential equation with nonconstant coefficients. The formal solution of this equation can be written

$$
P(t)=-\gamma \int_{-\infty}^{t} \exp \left[\gamma\left(t^{\prime}-t\right)\right] A\left(t^{\prime}\right) D\left(t^{\prime}\right) d t^{\prime},
$$

where it is again assumed that the polarization components vanish at the initial time $t=-\infty$. As discussed above, it is now helpful to replace the functions in the integrand by their Taylor series expansions in the vicinity of the time $t$, the effective peak of the $\delta$ function: 


$$
\begin{aligned}
A\left(t^{\prime}\right)= & A(t)+\frac{d A(t)}{d t}\left(t^{\prime}-t\right)+\frac{1}{2 !} \frac{d^{2} A(t)}{d t^{2}}\left(t^{\prime}-t\right)^{2} \\
& +\frac{1}{3 !} \frac{d^{3} A(t)}{d t^{3}}\left(t^{\prime}-t\right)^{3}+\cdots, \\
D\left(t^{\prime}\right)= & D(t)+\frac{d D(t)}{d t}\left(t^{\prime}-t\right)+\frac{1}{2 !} \frac{d^{2} D(t)}{d t^{2}}\left(t^{\prime}-t\right)^{2} \\
& +\frac{1}{3 !} \frac{d^{3} D(t)}{d t^{3}}\left(t^{\prime}-t\right)^{3}+\cdots .
\end{aligned}
$$

We will identify the terms in these series according to the power to which the term $\left(t^{\prime}-t\right)$ is raised. Thus, $A(t)$ is the zeroth-order term in the Taylor series expansion of $A\left(t^{\prime}\right)$ about the time $t$.

If the series in Eqs. (46) and (47) are substituted into Eq. (45), the formal solution for the polarization, one obtains the expansion

$$
\begin{aligned}
P(t)= & -\gamma \int_{-\infty}^{t} \exp \left[\gamma\left(t^{\prime}-t\right)\right]\left(A(t)+\frac{d A(t)}{d t}\left(t^{\prime}-t\right)\right. \\
& \left.+\frac{1}{2 !} \frac{d^{2} A(t)}{d t^{2}}\left(t^{\prime}-t\right)^{2}+\cdots\right)\left(D(t)+\frac{d D(t)}{d t}\left(t^{\prime}-t\right)\right. \\
& \left.+\frac{1}{2 !} \frac{d^{2} D(t)}{d t^{2}}\left(t^{\prime}-t\right)^{2}+\cdots\right) d t^{\prime} \\
= & -\gamma\left[A(t) D(t) \int_{-\infty}^{t} \exp \left[\gamma\left(t^{\prime}-t\right)\right] d t^{\prime}+\left(A(t) \frac{d D(t)}{d t}\right.\right. \\
& \left.+D(t) \frac{d A(t)}{d t}\right) \int_{-\infty}^{t} \exp \left[\gamma\left(t^{\prime}-t\right)\right]\left(t^{\prime}-t\right) d t^{\prime} \\
& +\left(\frac{1}{2 !} A(t) \frac{d^{2} D(t)}{d t^{2}}+\frac{d A(t)}{d t} \frac{d D(t)}{d t}\right. \\
& \left.+\frac{1}{2 !} D(t) \frac{d^{2} A(t)}{d t^{2}}\right) \int_{-\infty}^{t} \exp \left[\gamma\left(t^{\prime}-t\right)\right]\left(t^{\prime}-t\right)^{2} d t^{\prime} \\
& +\cdots] \cdot
\end{aligned}
$$

With the change of variables $x=\gamma\left(t-t^{\prime}\right)$, the integrals can be written more simply and Eq. (48) takes the form

$$
\begin{aligned}
P(t)= & -A(t) D(t) \int_{0}^{\infty} \exp (-x) d x+\frac{1}{\gamma}\left(A(t) \frac{d D(t)}{d t}\right. \\
& \left.+D(t) \frac{d A(t)}{d t}\right) \int_{0}^{\infty} x \exp (-x) d x \\
& -\frac{1}{\gamma^{2}}\left(\frac{1}{2 !} A(t) \frac{d^{2} D(t)}{d t^{2}}+\frac{d A(t)}{d t} \frac{d D(t)}{d t}\right. \\
& \left.+\frac{1}{2 !} D(t) \frac{d^{2} A(t)}{d t^{2}}\right) \int_{0}^{\infty} x^{2} \exp (-x) d x+\cdots
\end{aligned}
$$

The integrals in Eq. (49) can be evaluated using the formula [21]

$$
\int_{0}^{\infty} x^{n} \exp (-x) d x=n !,
$$

and the result of this substitution in Eq. (49) is

$$
\begin{aligned}
P(t)= & -A(t) D(t)+\frac{1}{\gamma}\left(A(t) \frac{d D(t)}{d t}+D(t) \frac{d A(t)}{d t}\right) \\
& -\frac{1}{\gamma^{2}}\left(A(t) \frac{d^{2} D(t)}{d t^{2}}+2 \frac{d A(t)}{d t} \frac{d D(t)}{d t}\right. \\
& \left.+D(t) \frac{d^{2} A(t)}{d t^{2}}\right)+\cdots .
\end{aligned}
$$

The first term in this expansion leads, of course, to the usual rate-equation approximation. We refer to this term alone as the zeroth-order rate-equation approximation, and it was derived for the more general laser model in the preceding section. The first two terms taken together represent the firstorder approximation, and so on. The higher-order terms in this expansion quickly lead to rather messy mathematics, and convergence of the overall process is not obvious. The firstorder approximation, however, is easy to work out in detail, and we will find that it can lead to a significant improvement in accuracy for a wide range of operating conditions when the polarization decay rate is large but not infinite.

If the first-order approximation for the polarization in Eq. (51) is substituted into the population and field equations given as Eqs. (43) and (44), one obtains the set

$$
\begin{aligned}
\frac{d D(t)}{d t}= & -\gamma_{d}\left[D(t)-r(t)+A^{2}(t) D(t)-\frac{A}{\gamma}\left(A(t) \frac{d D(t)}{d t}\right.\right. \\
& \left.\left.+D(t) \frac{d A(t)}{d t}\right)\right] \\
\frac{d A(t)}{d t} & =-\gamma_{c}\left[A(t)-A(t) D(t)+\frac{1}{\gamma}\left(A(t) \frac{d D(t)}{d t}\right.\right. \\
& \left.\left.+D(t) \frac{d A(t)}{d t}\right)\right]
\end{aligned}
$$

In terms of the normalized intensity $I(t)=A^{2}(t)$, these equations can also be written

$$
\begin{gathered}
\frac{d D(t)}{d t}=-\gamma_{d}\left(D(t)-r(t)+I(t) D(t)-\frac{I(t)}{\gamma} \frac{d D(t)}{d t}\right. \\
\left.-\frac{D(t)}{2 \gamma} \frac{d I(t)}{d t}\right), \\
\frac{d I(t)}{d t}=-2 \gamma_{c}\left(I(t)-I(t) D(t)+\frac{I(t)}{\gamma} \frac{d D(t)}{d t}+\frac{D(t)}{2 \gamma} \frac{d I(t)}{d t}\right) .
\end{gathered}
$$


It is clear from the form of Eqs. (54) and (55) that the derivative terms on the right-hand side will drop out in the usual zeroth-order rate-equation limit, in which the coherence decay rate approaches infinity. Retaining these terms, there are still at least two reasonable approaches that one could take in working toward solutions. The simplest idea would be to replace the derivatives on the right-hand side of the equations with their zeroth-order approximations (from the same equations with $\gamma=\infty$ ). This apparent further approximation is not necessary, however, and instead we group the derivatives to the left-hand sides of the equations, yielding

$$
\begin{gathered}
\left(-\frac{1}{\gamma_{d}}+\frac{I(t)}{\gamma}\right) \frac{d D(t)}{d t}+\frac{D(t)}{2 \gamma} \frac{d I(t)}{d t}=D(t)-r(t) \\
+I(t) D(t) \\
-\frac{I(t)}{\gamma} \frac{d D(t)}{d t}+\left(-\frac{1}{2 \gamma_{c}}-\frac{D(t)}{2 \gamma}\right) \frac{d I(t)}{d t}=I(t)-I(t) D(t)
\end{gathered}
$$

Equations (56) and (57) may be regarded as two equations in two unknowns for the derivatives of the population and the intensity. They may be readily solved for these derivatives and the results are

$$
\begin{gathered}
\frac{d D(t)}{d t}=\frac{-\gamma_{d}\left\{[D(t)-r(t)+I(t) D(t)]-\frac{\gamma_{c}}{\gamma}[r(t)-I(t)-D(t)] D(t)\right\}}{1-\frac{\gamma_{d}}{\gamma} I(t)+\frac{\gamma_{c}}{\gamma} D(t)}, \\
\frac{d I(t)}{d t}=\frac{-2 \gamma_{c} I(t)\left\{[1-D(t)]+\frac{\gamma_{d}}{\gamma}[r(t)-I(t)-D(t)]\right\}}{1-\frac{\gamma_{d}}{\gamma} I(t)+\frac{\gamma_{c}}{\gamma} D(t)} .
\end{gathered}
$$

We refer to Eqs. (58) and (59) as the first-order rateequations, and higher-order forms can be developed in a similar way by retaining higher-order derivatives in the various expansions. The zeroth-order limit is obtained from these equations when the polarization decay rate is much larger than both the population and field decay rates. For reference, we also write the familiar zeroth-order equations explicitly here:

$$
\begin{gathered}
\frac{d D(t)}{d t}=-\gamma_{d}[D(t)-r(t)+I(t) D(t)], \\
\frac{d I(t)}{d t}=-2 \gamma_{c} I(t)[1-D(t)] .
\end{gathered}
$$

These forms are equivalent to the rate equations of Statz and DeMars [2] and Dunsmuir [3]. To this level of approximation it is possible that other arrangements of the correction terms in Eqs. (58) and (59) would be equally accurate. For example, one could imagine moving the corrections in the denominators into the numerators on the basis that they might be small compared to unity. The advantages of such potential rearrangements are not very obvious, and we do not consider them further.

\section{NUMERICAL SOLUTIONS}

Except in very special cases, it proves to be difficult to find analytic solutions to any of the dynamical equations developed above. Numerical solutions, however, are always possible. In this section, we examine numerically some of the implications of the three models described above. These include the simplest possible three-equation model for the homogeneously broadened laser, which we will call the exact model, and the two lowest-level rate-equation models.

One difficulty in determining the general behavior possibilities for the laser models is the number of independent parameters that they contain. In Eqs. (42)-(44), for example, one encounters the polarization decay rate $\gamma$, the population decay rate $\gamma_{d}$, the field amplitude or cavity decay rate $\gamma_{c}$, and the threshold parameter $r(t)$. It is convenient to absorb one of the decay rates into a normalization of the time variable; and, as in a study of laser instabilities, we introduce the new time constant $\tau=\gamma_{c} t$ [20]. With this change of variables, Eqs. (42)-(44) can be written

$$
\begin{gathered}
\frac{d P(\tau)}{d \tau}=-\delta[P(\tau)+A(\tau) D(\tau)], \\
\frac{d D(\tau)}{d \tau}=-\delta \rho[D(\tau)-r(\tau)-A(\tau) P(\tau)], \\
\frac{d A(\tau)}{d \tau}=-[A(\tau)+P(\tau)],
\end{gathered}
$$

where we have also introduced the decay-rate ratios $\rho=\gamma_{d} / \gamma$ and $\delta=\gamma / \gamma_{c}$ [20]. With these normalizations the equations now have only three parameters: $\delta, \rho$, and $r$.

The two rate-equation approximations can be simplified in a similar way. Thus, the first-order rate-equation model given in Eqs. (58) and (59) can be written 


$$
\frac{d D(\tau)}{d \tau}=\frac{-\delta \rho\{[D(\tau)-r(\tau)+I(\tau) D(\tau)]-[r-I(\tau)-D(\tau)] D(\tau) / \delta\}}{1-\rho I(\tau)+D(\tau) / \delta},
$$

$$
\frac{d I(\tau)}{d \tau}=\frac{-2 I(\tau)\{[1-D(\tau)]+\rho[r-I(\tau)-D(\tau)]\}}{1-\rho I(\tau)+D(\tau) / \delta} .
$$

Similarly, the zeroth-order approximation follows from Eqs. (60) and (61):

$$
\begin{gathered}
\frac{d D(\tau)}{d \tau}=-\delta \rho[D(\tau)-r(\tau)+I(\tau) D(\tau)], \\
\frac{d I(\tau)}{d \tau}=-2 I(\tau)[1-D(\tau)] .
\end{gathered}
$$

The most straightforward way to compare the three models that have just been summarized is to graphically superimpose their predictions for the same set of conditions. The rate-equation approximations are only expected to be useful for relatively large values of the coherence decay rate $\gamma$. In the simplified notation employed above, it is clear that these models should be compared especially for the cases $\delta \gg 1$ and $\rho \ll 1$. Figure 1 includes plots of the response of a prepumped laser when a small initial field amplitude $(A=0.01)$ is introduced according to each of the three models. The decay-rate ratios in this case have the intermediate values $\delta=10$ and $\rho=0.1$. In Fig. 1(a), the threshold parameter has the modest
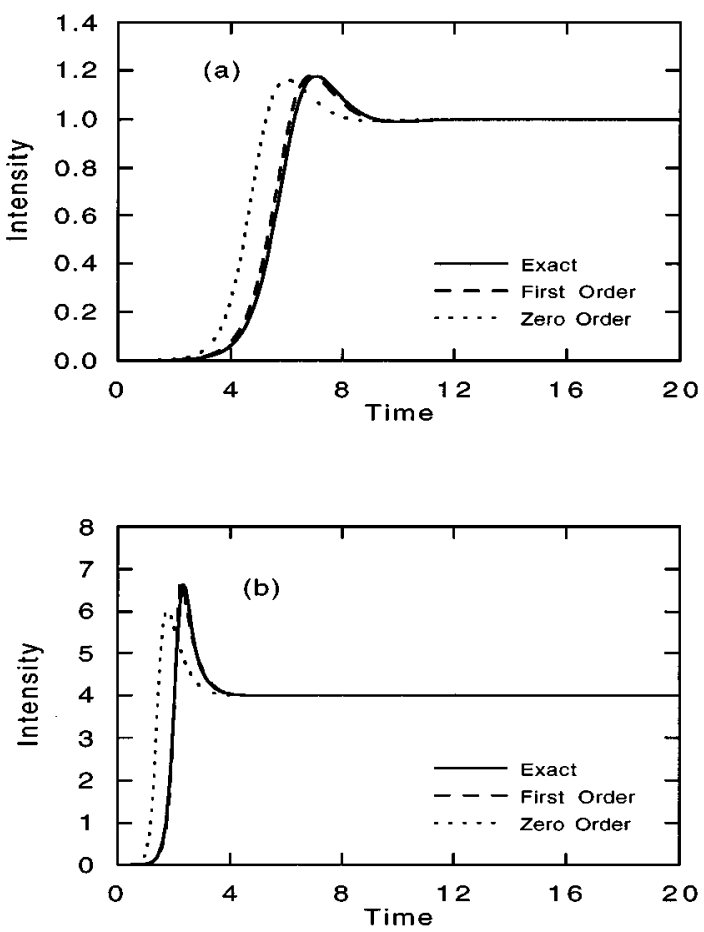

FIG. 1. Intensity as a function of time in a prepumped laser having the decay rate ratios $\rho=\gamma_{d} / \gamma=0.1$ and $\delta=\gamma / \gamma_{c}=10$. In (a) the threshold parameter is $r=2$ and in (b) it is $r=5$. For most practical operating conditions, the first-order rate-equation approximation provides much better agreement with the exact model than does the usual zeroth-order approximation. practical value $r=2$, and it can be seen from the figure that in this case the output consists of a single pulse that decays directly to the steady-state output value $I=1$. This operating state might be thought to lie well within the usual rateequation conditions, and it can be seen from the figure that the exact model and the first-order rate-equation model are almost indistinguishable. The only noticeable difference is that the initial pulse is just slightly early in the first-order rate-equation case.

In contrast to the first-order case, the zeroth-order rateequation limit involves much more substantial errors. The output pulse is significantly early and slightly small compared to the exact model. Figure 1(b) repeats the same calculations, but with the higher threshold parameter value $r=5$. The general differences between the models are continued for this higher pump rate, but the pulses generally are earlier and larger. For very large and less practical pump rates the differences between the models increase. With these decay-rate ratios, the first-order model ultimately leads to implausible behavior because the correction terms may cease to be small or may change sign. The zeroth-order model, on the other hand, continues to look plausible but in fact also departs dramatically from the exact solution.

To provide further insight into the agreements and discrepancies between the models, other numerical solutions are
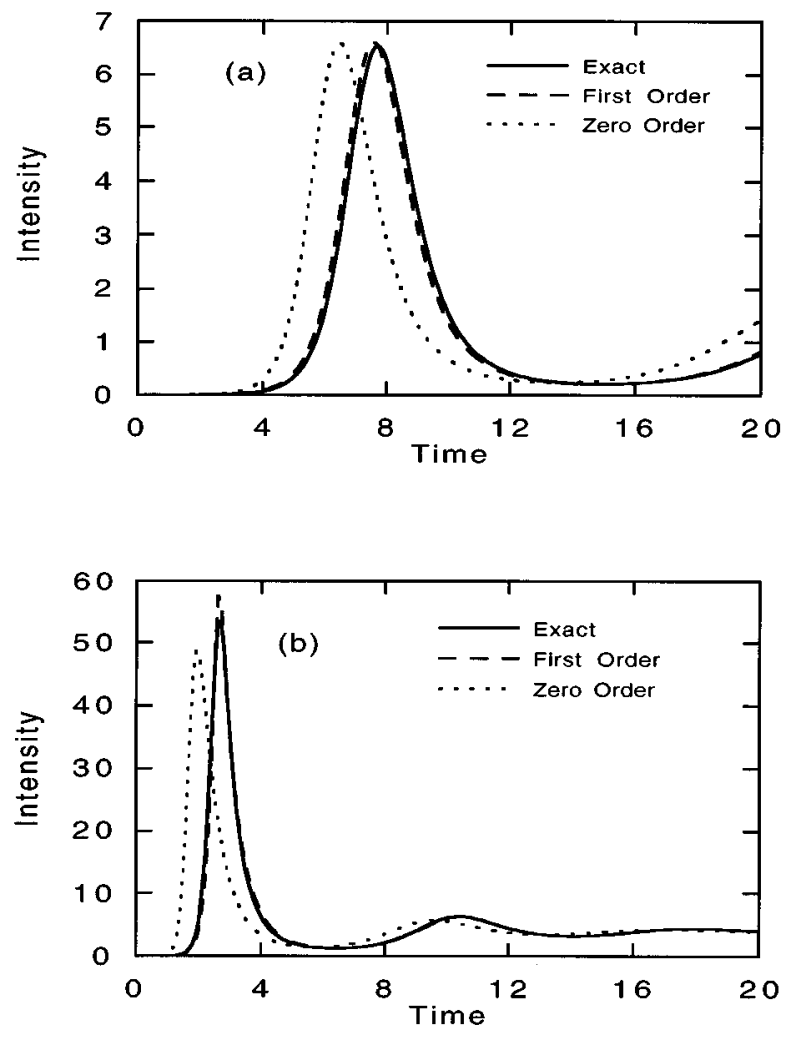

FIG. 2. Intensity as a function of time in a prepumped laser having the decay rate ratios $\rho=0.01$ and $\delta=10$. In (a) the threshold parameter is $r=2$ and in (b) it is $r=5$. 

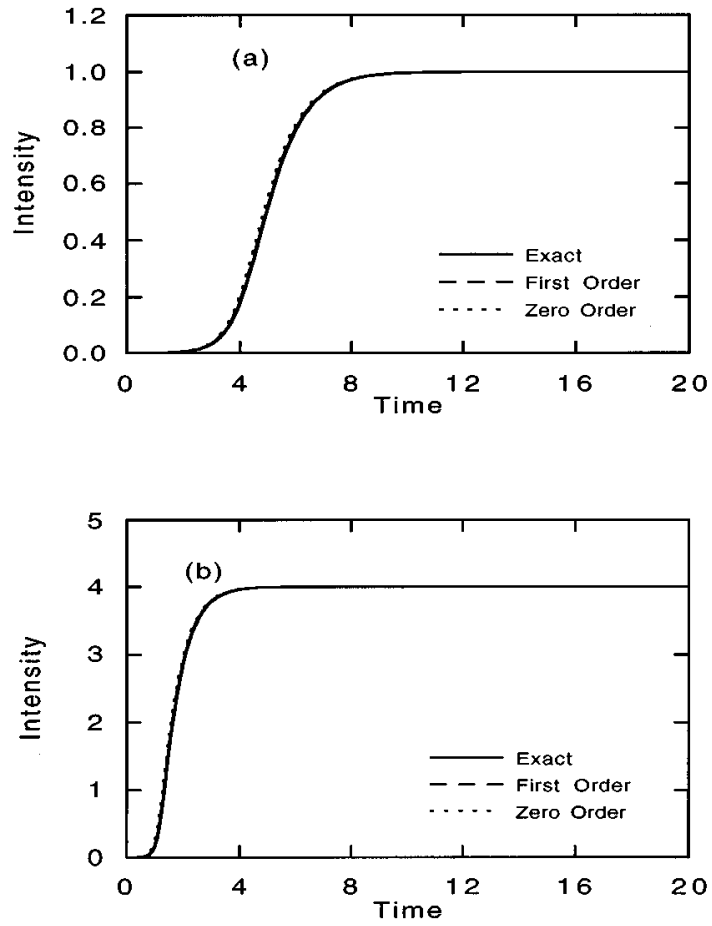

FIG. 3. Intensity as a function of time in a prepumped laser having the decay rate ratios $\rho=0.1$ and $\delta=100$. In (a) the threshold parameter is $r=2$ and in (b) it is $r=5$. In this limit, both approximations agree well with the exact solutions.

presented in Figs. 2 and 3. In Fig. 2 the decay-rate ratios have the values $\delta=10$ and $\rho=0.01$, and the threshold parameter again takes on the values $r=2$ [Fig. 2(a)] and $r=5$ [Fig. 2(b)]. In these cases, the response of the laser might be characterized as a giant pulse followed by damped relaxation oscillations. The first-order rate-equation approximation again agrees very closely with the exact solution, while the shortcomings of the zeroth-order model in pulse timing and height are conspicuous. In Fig. 3 the decay-rate ratios have the values $\delta=100$ and $\rho=0.1$, and the threshold parameter again takes on the values $r=2$ and $r=5$. In these cases, the response is so strongly damped that no pulse forms, and it may be seen that both of the rate-equation approximations are in close agreement with the exact model.

\section{LINEARIZATION}

As noted above, numerical methods are required to solve the nonlinear models of interest here for arbitrary values of the various parameters. However, an extremely useful approximation is obtained by restricting attention to a region very close to a steady-state operating point. In this case, the equations can be linearized, and solutions become much more straightforward. Although this method is usually applied to homogeneously broadened media, it can also be employed with inhomogeneous broadening [22].

The linearization of Eqs. (42)-(44) is obtained by means of the substitutions $P(t)=P_{1}+P^{\prime}(t), D(t)=D_{1}+D^{\prime}(t)$, and $A(t)=A_{1}+A^{\prime}(t)$, where the primed perturbation quantities are assumed to always remain very small compared to the corresponding subscripted steady-state values. If the quadratic terms in the primed quantities are assumed to be neg-
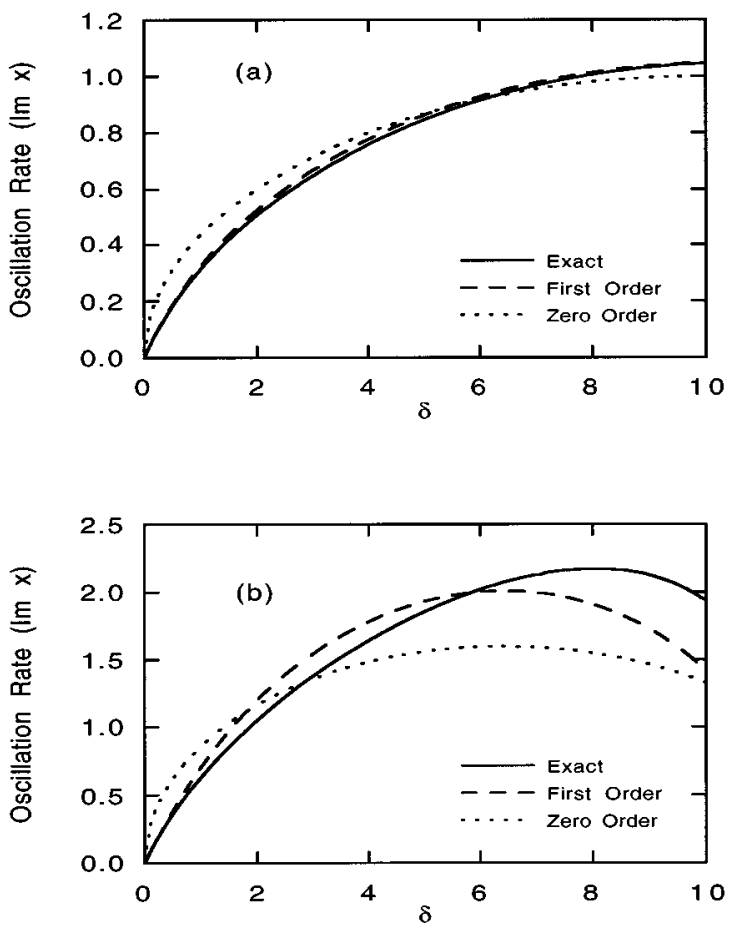

FIG. 4. Oscillation frequency as a function of $\delta$ for $\rho=0.1$. In (a) the threshold parameter is $r=2$ and in (b) it is $r=5$. The first-order approximation is more accurate than the zeroth-order approximation, especially for smaller values of the threshold parameter.

ligible, these substitutions reduce Eqs. (42)-(44) to the linear set

$$
\begin{gathered}
\frac{d P^{\prime}(t)}{d t}=-\gamma\left[P^{\prime}(t)+A_{1} D^{\prime}(t)+A^{\prime}(t) D_{1}\right] \\
\frac{d D^{\prime}(t)}{d t}=-\gamma_{d}\left[D^{\prime}(t)-A_{1} P^{\prime}(t)-A^{\prime}(t) P_{1}\right] \\
\frac{d A^{\prime}(t)}{d t}=-\gamma_{c}\left[A^{\prime}(t)+P^{\prime}(t)\right] .
\end{gathered}
$$

The associated steady-state solutions are $P_{1}=-A_{1}, D_{1}=1$, and $A_{1}^{2}=r-1$.

The advantage of linear equations, of course, is that the solutions are always in the form of exponentials. Thus one is led to try solutions of the form $P^{\prime}(t)=P^{\prime \prime} \operatorname{Re}[\exp (s t)]$, $D^{\prime}(t)=D^{\prime \prime} \operatorname{Re}[\exp (s t)]$, and $A^{\prime}(t)=A^{\prime \prime} \operatorname{Re}[\exp (s t)]$, where Re denotes the real part. The results of these substitutions are the algebraic equations

$$
\begin{gathered}
s P^{\prime \prime}=-\gamma\left(P^{\prime \prime}+A_{1} D^{\prime \prime}+A^{\prime \prime} D_{1}\right), \\
s D^{\prime \prime}=-\gamma_{d}\left(D^{\prime \prime}-A_{1} P^{\prime \prime}-A^{\prime \prime} P_{1}\right), \\
s A^{\prime \prime}=-\gamma_{c}\left(A^{\prime \prime}+P^{\prime \prime}\right) .
\end{gathered}
$$

These equations can be combined with the steady-state results to obtain a cubic equation for the complex eigenvalue $s$ :

$$
s^{3}+\left(\gamma+\gamma_{c}+\gamma_{d}\right) s^{2}+\gamma_{d}\left(\gamma_{c}+r \gamma\right) s+2(r-1) \gamma \gamma_{c} \gamma_{d}=0 \text {. }
$$



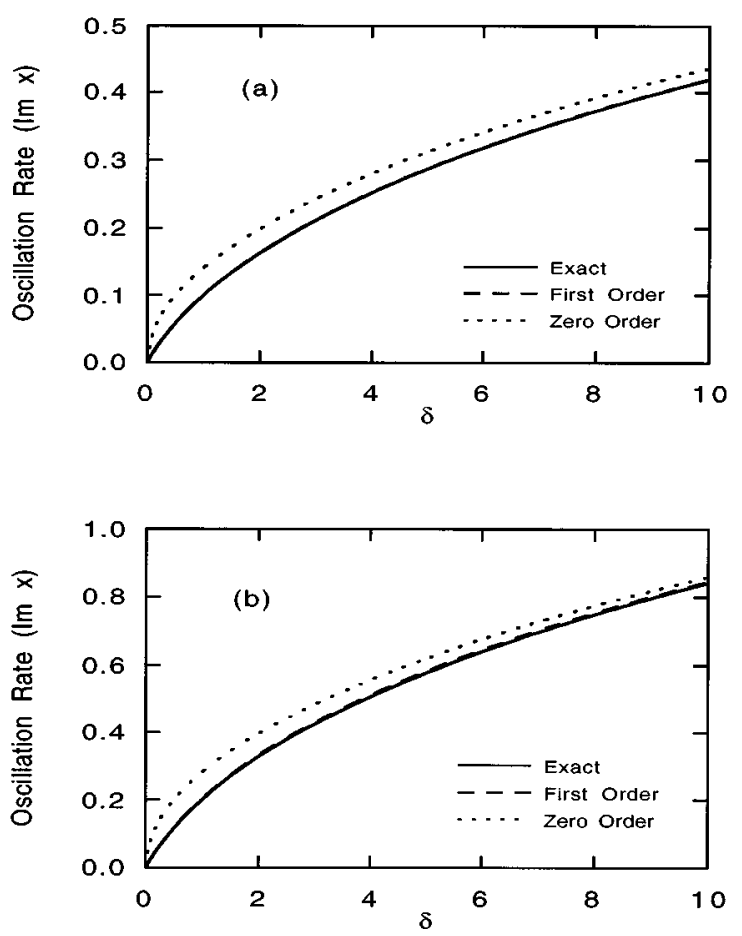

FIG. 5. Oscillation frequency as a function of $\delta$ for $\rho=0.01$. In (a) the threshold parameter is $r=2$ and in (b) it is $r=5$. The firstorder approximation is almost indistinguishable from the exact solution for both values of the threshold parameter.

The rate-equation models can also be linearized and solved. Thus, the linearization of the first-order model given in Eqs. (58) and (59) is also obtained by means of the substitutions $D(t)=D_{1}+D^{\prime}(t)$, and $A(t)=A_{1}+A^{\prime}(t)$. If the quadratic terms in the small quantities are neglected, these equations become

$$
\frac{d D^{\prime}(t)}{d t}=\frac{-\gamma_{d}\left\{r D^{\prime}(t)+I^{\prime}(t)+\left(\gamma_{c} / \gamma\right)\left[D^{\prime}(t)+I^{\prime}(t)\right]\right\}}{1-\left(\gamma_{d} / \gamma\right)(r-1)+\left(\gamma_{c} / \gamma\right)},
$$

$$
\frac{d I^{\prime}(t)}{d t}=\frac{-2 \gamma_{c}(r-1)\left\{-D^{\prime}(t)-\left(\gamma_{d} / \gamma\right)\left[D^{\prime}(t)+I^{\prime}(t)\right]\right\}}{1-\left(\gamma_{d} / \gamma\right)(r-1)+\left(\gamma_{c} / \gamma\right)},
$$

where the steady-state results $D_{1}=1$ and $I_{1}=r-1$ have already been introduced.

One is again led to try solutions of the form $D^{\prime}(t)=D^{\prime \prime} \operatorname{Re}[\exp (s t)]$ and $I^{\prime}(t)=I^{\prime \prime} \operatorname{Re}[\exp (s t)]$, and the results of these substitutions in Eqs. (76) and (77) are

$$
\begin{aligned}
& s D^{\prime \prime}=\frac{-\gamma_{d}\left[r D^{\prime \prime}+I^{\prime \prime}+\left(\gamma_{c} / \gamma\right)\left(D^{\prime \prime}+I^{\prime \prime}\right)\right]}{1-\left(\gamma_{d} / \gamma\right)(r-1)+\left(\gamma_{c} / \gamma\right)}, \\
& s I^{\prime \prime}=\frac{2 \gamma_{c}(r-1)\left[D^{\prime \prime}+\left(\gamma_{d} / \gamma\right)\left(D^{\prime \prime}+I^{\prime \prime}\right)\right]}{1-\left(\gamma_{d} / \gamma\right)(r-1)+\left(\gamma_{c} / \gamma\right)} .
\end{aligned}
$$

These equations can be combined to obtain a quadratic equation for the complex eigenvalue $s$ :
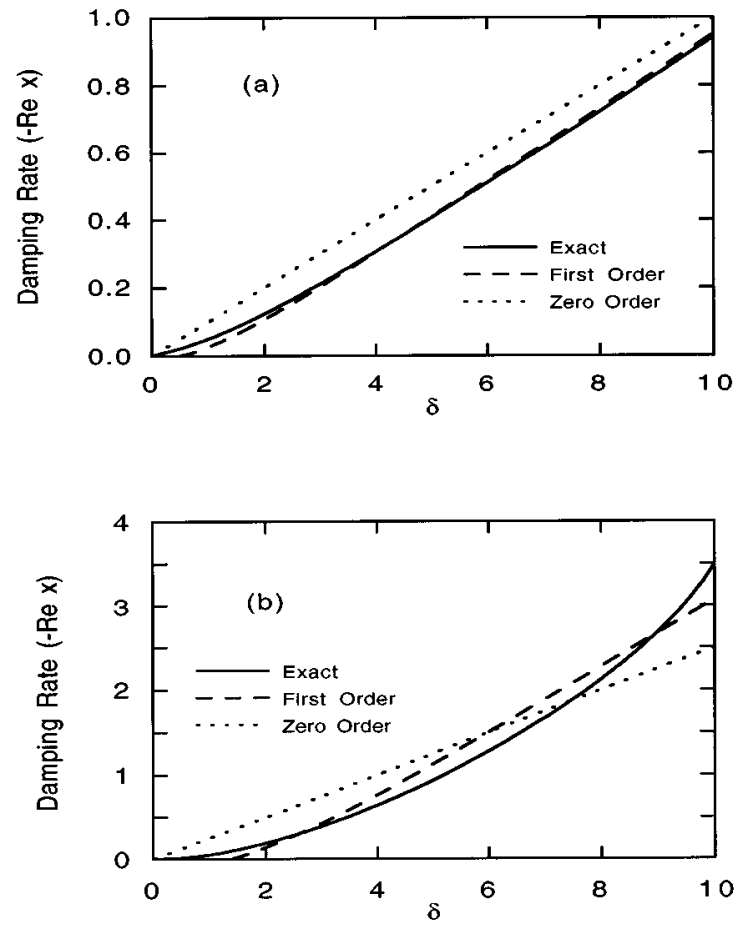

FIG. 6. Damping rate as a function of $\delta$ for $\rho=0.1$. In (a) the threshold parameter is $r=2$ and in (b) it is $r=5$. The first-order approximation is more accurate than the zeroth-order approximation, especially for smaller values of the threshold parameter.

$$
\begin{aligned}
& \left(1-\frac{\gamma_{d}}{\gamma}(r-1)+\frac{\gamma_{c}}{\gamma}\right) s^{2}+\gamma_{d}\left[r\left(1-\frac{2 \gamma_{c}}{\gamma}\right)+\frac{3 \gamma_{c}}{\gamma}\right] s \\
& +2 \gamma_{c} \gamma_{d}(r-1)=0 .
\end{aligned}
$$

In the zeroth-order approximation, the decay-rate ratios in Eq. (80) vanish, and the complex eigenvalues are solutions of the familiar equation

$$
s^{2}+\gamma_{d} r s+2 \gamma_{c} \gamma_{d}(r-1)=0
$$

In summary, we now have three different equations for the complex oscillation frequency $s$. These include Eq. (75) for our so-called exact solutions, Eq. (80) for our first-order rate-equation approximation, and Eq. (81) for the standard zeroth-order rate-equation approximation. As with the numerical solutions discussed above, it is convenient to absorb the field decay rate into the complex oscillation frequency by means of the substitution $x=s / \gamma_{c}$. With this substitution, Eqs. (75), (80), and (81) reduce, respectively, to the simpler forms

$$
x^{3}+(1+\delta+\rho \delta) x^{2}+\rho \delta(1+r \delta) x+2 \rho \delta^{2}(r-1)=0,
$$

$$
\begin{gathered}
{[1-\rho(r-1)+1 / \delta] x^{2}+\rho \delta[r(1-2 / \delta)+3 / \delta] x+2 \rho \delta(r-1)} \\
=0, \\
x^{2}+\rho \delta r x+2 \rho \delta(r-1)=0,
\end{gathered}
$$

where our previous decay-rate ratios have also been employed. 

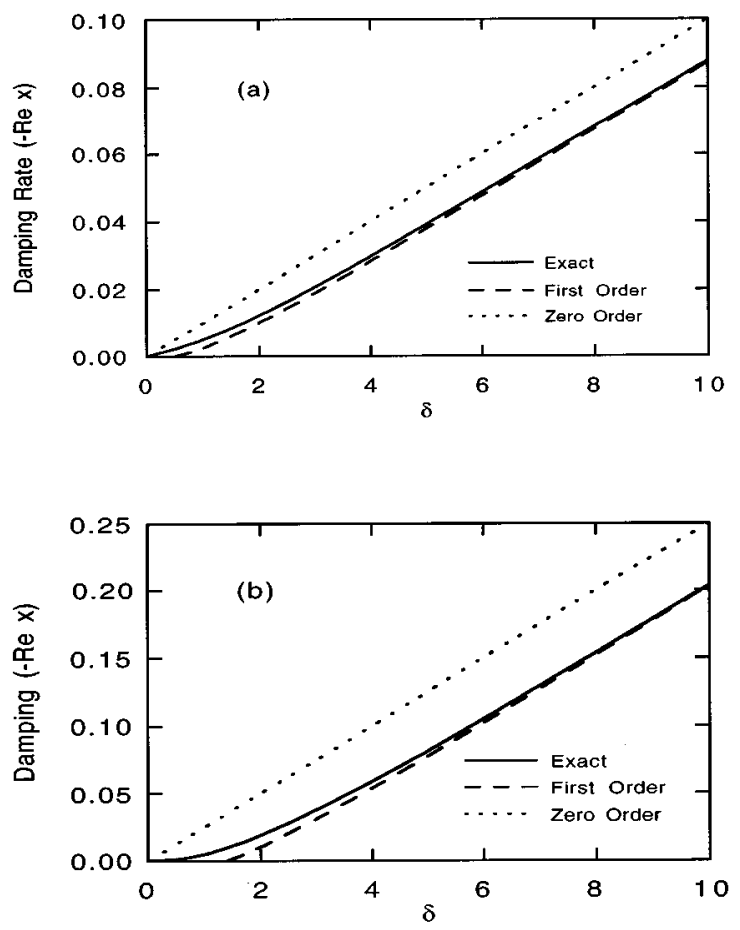

FIG. 7. Damping rate as a function of $\delta$ for $\rho=0.01$. In (a) the threshold parameter is $r=2$ and in (b) it is $r=5$. The first-order approximation is almost indistinguishable from the exact solution for both values of the threshold parameter and $\delta$ greater than 2 or 3 .

Equations (82)-(84) may be solved analytically using well known methods [23]. The real and imaginary parts of the complex frequency $x$ are proportional to the damping and the frequency of small amplitude relaxation oscillations, respectively [22]. The oscillation frequency for the three models is plotted in Fig. 4 as a function of the lifetime ratio $\delta$ for $\rho=0.1$. In Fig. 4(a) the threshold parameter has the modest value $r=2$, and it can be seen that the first-order and the exact solutions are in very good agreement for all values of $\delta$. This is a better result than one might have anticipated, since it is only for large values of $\delta$ that our derivation of the first-order approximation would seem to be valid. In general, the zeroth-order result is not nearly as accurate. In Fig. 4(b) the same calculations are done but for the larger threshold parameter value $r=5$. In this case, the approximations may only be said to provide a qualitative description of the oscillation frequency. The approximations improve as the value of $\rho$ is decreased. As shown in Fig. 5, with $\rho=0.01$ the firstorder approximation is almost indistinguishable from the exact solution for all values of $\delta$ and for both values of the threshold parameter. On the other hand, even in this case the ordinary zeroth-order approximation is seen to lead to substantial errors in estimating the relaxation oscillation frequency.

In a similar way, the real part of the complex oscillation frequency can be obtained to study the damping rate of the relaxation oscillations. The damping rate for the three models is plotted in Fig. 6 as a function of the lifetime ratio $\delta$ for $\rho=0.1$. In Fig. 6(a) the threshold parameter again has the value $r=2$, and it can be seen that the first-order model provides good results down to $\delta$ values of about two or three.
Again, the usual zeroth-order result is not nearly so good. In Fig. 6(b) the calculations are repeated for the larger threshold parameter value. In this case, the approximations are again only qualitatively accurate. For smaller values of $\rho$ the range of usefulness of the first-order approximation is greatly increased. As shown in Fig. 7, with $\rho=0.01$ this approximation is almost indistinguishable from the exact solution for values of $\delta$ greater than two or three for both values of the threshold parameter. The zeroth-order approximation is not nearly as accurate.

\section{CONCLUSION}

Many methods have been developed for analyzing the dynamical behavior of lasers. Among the more rigorous of these are the so-called semiclassical models, which are based directly on the Maxwell-Heaviside and Schrödinger equations. While these rigorous models are necessary for the investigation of some important laser phenomena, it is often possible and desirable to use more simplified formulations. One of the best known of these simplifications is the socalled rate-equation approximation. In this approximation, the phase relaxation rate of the atomic or molecular wave functions is assumed to be much larger than any other rate of interest, and thus the polarization amplitude associated with the wave functions can be regarded as an instantaneous function of the electromagnetic field amplitude and population variables. In this study the range of validity of the usual rate-equation approximation has been explored in detail and a more accurate rate-equation model has been developed. In this model, the polarization is still eliminated as a dynamical variable, but the coherence decay rate is retained in the equations. It has been shown here that this intermediate formulation is only slightly more complicated than the usual rateequation model while providing dramatically improved accuracy for most practical laser operating conditions.

The model reduction procedure described here should be useful in other applications as well. It may be of interest for any situation in which one variable is adiabatically eliminated from a dynamical model. In the laser context again, it may be noted that for some lasers it makes sense to consider eliminating the electric-field amplitude on the basis that the field decay rate is faster than the polarization and population decay rates. Although not so common as the rate-equation approximation, this situation has also long been recognized [10]. Using the methods described here, useful intermediate results for this case can readily be obtained.

\section{ACKNOWLEDGMENTS}

This work was supported in part by the National Science Foundation under Grant No. PHY94-15583. The author also expresses his appreciation to J. H. Eberly, D. G. Hall, and other members of the Rochester Theory Center for Optical Science and Engineering and The Institute of Optics at the University of Rochester for valuable discussions and hospitality during his sabbatical visit. 
[1] A. Einstein, Mitteilungen der Physikalischen Gesellschaft Zürich, 16, 47 (1916); Physickal. Z. 18, 121 (1917) [translated into English in Sources of Quantum Mechanics, edited by B. L. van der Waerden (North-Holland, Amsterdam, 1967)], pp. 63-77; in The World of the Atom, edited by H. A. Boorse and L. Motz (Basic Books, New York, 1966), Vol. 2, pp. 888-901; in The Old Quantum Theory, edited by D. ter Haar (Pergamon, New York, 1967), pp. 167-183].

[2] H. Statz and G. DeMars, in Quantum Electronics, A Symposium, edited by C. H. Townes (Columbia University Press, New York, 1960), pp. 530-537.

[3] R. Dunsmuir, J. Electron. Control 10, 453 (1961).

[4] J. S. Wright and E. O. Schulz-DuBois, Solid-State Maser Research, Fifth Quarterly Report, U.S. Army Signal Corps Contract No. DA36-039SC-85357, 1961 (unpublished) (available through Armed Services Technical Information Agency, Defense Documentation Center as No. Ad 265838.

[5] W. W. Rigrod, J. Appl. Phys. 34, 2602 (1963).

[6] R. Bellman, G. Birnbaum, and W. G. Wagner, J. Appl. Phys. 34, 780 (1963).

[7] L. M. Frantz and J. S. Nodvik, J. Appl. Phys. 34, 2346 (1963).

[8] E. O. Schulz-DuBois, Bell Syst. Tech. J. 43, 625 (1964).

[9] A. G. Gurtovnik, Izv. Vyssh. Uchebn. Zaved. Radiofiz. 1, 83 (1958) [English translation TG230-T382 in Applied Physics
Laboratory Library Bulletin (Johns Hopkins University, Baltimore, 1963)].

[10] C. L. Tang, J. Appl. Phys. 34, 2935 (1963).

[11] W. E. Lamb, Jr., Phys. Rev. 134, A1429 (1964).

[12] L. W. Casperson, J. Opt. Soc. Am. B 5, 958 (1988), and references therein.

[13] L. W. Casperson, Phys. Rev. A 42, 6721 (1990).

[14] L. W. Casperson, Phys. Rev. A 43, 5057 (1991).

[15] L. W. Casperson, Phys. Rev. A 44, 3291 (1991), and references therein.

[16] L. W. Casperson, Phys. Rev. A 46, 401 (1992).

[17] W. Kaplan, Ordinary Differential Equations (Addison-Wesley, Palo Alto, CA, 1962), Eq. (2-58).

[18] L. A. Lugiato, P. Mandel, and L. M. Narducci, Phys. Rev. A 29, 1438 (1984).

[19] See, for example, N. G. de Bruijn, Asymptotic Methods in Analysis (North-Holland, Amsterdam, 1970), Chap. 4.

[20] L. W. Casperson, J. Opt. Soc. Am. 2, 993 (1985).

[21] C.R.C. Standard Mathematical Tables, 12th ed., edited by C. D. Hodgman (Chemical Rubber Publishing Company, Cleveland, Ohio, 1961), p. 313.

[22] L. W. Casperson and A. Yariv, IEEE J. Quantum Electron. QE-8, 69 (1972).

[23] C.R.C. Standard Mathematical Tables (Ref. [21]), p. 358. 